\title{
Parallel-Connected Legs in a Grid-Tied Inverter System for Distributed Generation
}

\author{
G.J. Capellá ${ }^{1}$ J. Pou ${ }^{1}$, J. Zaragoza ${ }^{1}$, S. Ceballos ${ }^{2}$, I. Gabiola ${ }^{2}$, and E. Robles ${ }^{2}$ \\ ${ }^{1}$ Department of Electronical Engineering \\ Technical University of Catalonia. \\ Campus of Terrassa, 08222 Terrassa (Spain) \\ Phone:+0034 937398306, e-mail: pou@eel.upc.edu, capella@eel.upc.edu, zaragoza@eel.upc.edu \\ ${ }^{2}$ Tecnalia Technology Corporation, Energy Unit \\ Zamudio, 48170 Zamudio-Bizkaia (Spain) \\ Phone:+0034 944041444, e-mail: salvador.ceballos@tecnalia.com, igor.gabiola@tecnalia.com, \\ eider.robles@tecnalia.com
}

\begin{abstract}
Grid-connected high-power inverters are often used in distributed generation and power quality systems. Those inverters have to handle high currents in order to achieve high power values without resorting to higher voltages. Connecting inverter legs in parallel is a proper way to achieve such high currents. Such parallel connection is made by means of inductors and achieving balanced currents among the legs becomes a critical issue. Circulating currents may produce additional losses and stress to the converter's power devices. Therefore, they should be controlled and minimized. An efficient technique to achieve such balance is presented in this paper. The proposed strategy has been developed on a gridconnected three-phase system. Each phase of the inverter is made up of three legs in parallel. Besides, the control implemented in this work allows full regulation of the power factor. This way, any desired power factor value can be achieved in order to meet grid-connection requirements. Simulation and experimental results are shown in this paper.
\end{abstract}

\section{Key words}

Grid-connected converter, Distributed generation, Converter Control, Pulsewidth Modulation, Parallel legs.

\section{Introduction}

Significant electrical grid changes are being produced nowadays. Distributed generation systems are becoming more and more common; thus, huge central stations are starting to share electricity production with such distributed systems. Many of those generation devices require to process voltages and currents through power electronic converters for a proper grid connection. Some examples include renewable energy systems; e. g. solar photovoltaic, wind turbines, and marine energy. Other promising generation sources are fuel cells. Some other systems that require grid-connected converters are storage energy and power quality devices, such as active filters, static compensators (STATCOMs), dynamic voltage restorers (DVRs), etc.

In order to increase the rated power of power electronic converters, either the voltages or the currents can be increased (or both of them). Multilevel inverters are based on increasing the voltages handled by the converter. The main reason for this is because they can deal with higher voltages.

On the other hand, paralleling devices, legs, or converters is a way to increase the current values [1]-[5]. Parallelconnected legs of a voltage-source inverter (VSI) require the use of inductors to obtain a single output voltage from several input legs. It would be optimal if current sharing among the legs was balanced; however, there is no guaranty for this unless a proper control is used. Several techniques can be applied to achieve current balance among the legs. Most of them are based on PIs [3]-[5], [7] or optimal controllers [8] which can usually provide good balancing performance. However, they need parameter tuning and the balancing dynamic may not be optimal. Quick response of the balancing control is crucial to avoid long transitory overcurrents on specific legs which might be destructive. The balancing strategy used in this paper is based on [9] and it can achieve current balance very quickly since the exact modification of the modulation signals is calculated and applied. The method is performed without distorting the output voltages and currents.

In voltage-source inverters (VSIs), if the dc-link is fed by a current source, the converter itself takes care of the dclink voltage regulation. Therefore, a proper control loop is needed. In this work, a voltage-oriented control (VOC) is used [10],[11].

This paper is organized as follows. Firstly, an introduction to paralleling legs by means of inductors is made. Then, a three-phase parallel-legged grid-connected system is introduced. In the following sections, the current balancing technique used in this application is presented and the control loop is described. Finally, some simulation and experimental results are presented

\section{The grid-connected system}

The core of the plant used to test the control strategy is a grid-connected inverter whose phases are made up of three parallel legs (Fig. 1). The primary source of energy is represented by a current source. This current could come from different kinds of applications, such as 


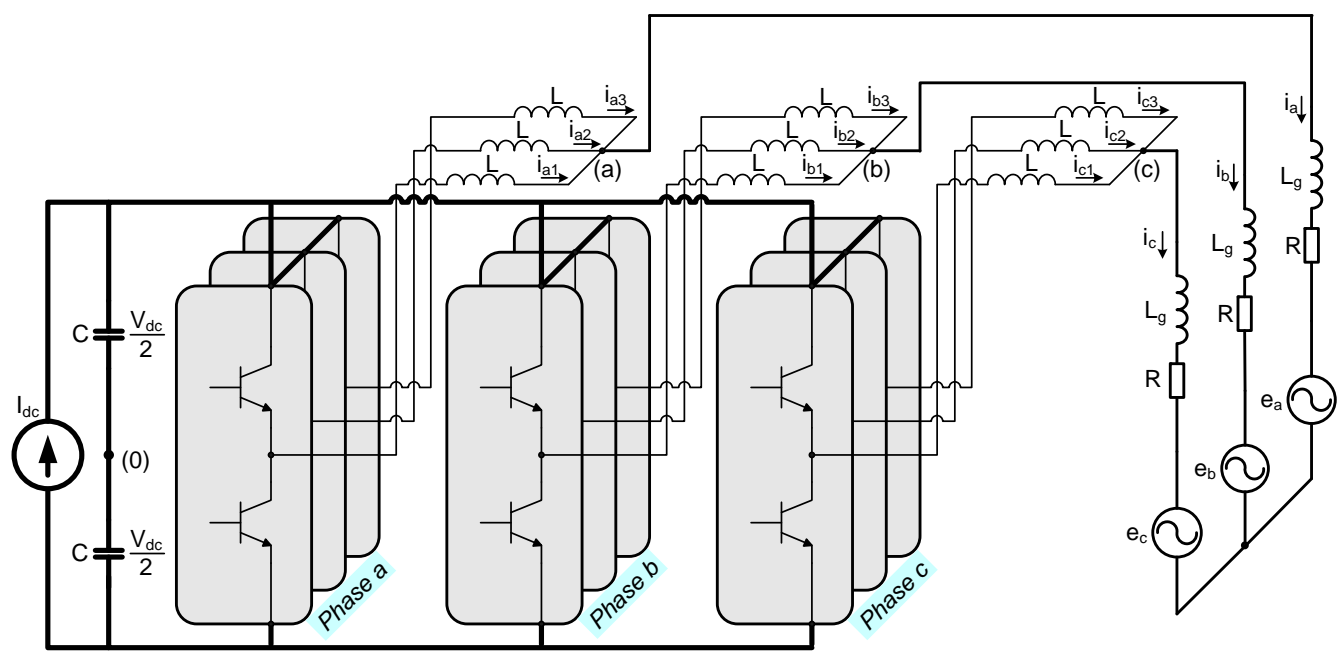

Fig. 1. Grid-connected structure

photovoltaic panels, wind turbines, or storage energy systems. The dc-link voltage is regulated by a proper control loop.

Inductors are used in order to restrain circulating currents among the legs of the same phase $(L)$. Those inductors also help to average the phase voltages coming from every paralleled leg. Additional inductances may be needed for the grid connection. Inductances $L_{g}$ stand for both, the intrinsic grid impedances and those additional inductances.

\section{Current balancing method}

In this study, no magnetic coupling among the inductors is assumed; therefore, the equivalent output inductance is $L / 3$. Consequently, the same inductances used for the parallel connection among the legs contribute to the output inductance of the phase needed in grid-connected applications.

An efficient current balancing strategy was shown in [9] to connect a paralleled-leg inverter over a passive load. The same strategy is applied to the grid-connected threephase system studied in this paper. A summary of the main concepts and equations used are given in the following.

The relationship between voltages and currents in each leg of the system in Fig. 1 is

$$
L \frac{d i_{x y}}{d t}=v_{x y}-v_{x} ; \text { for } x=\{a, b, c\} \text { and } y=\{1,2,3\} .
$$

Adding up the terms for each phase $(x)$, and taking into account that $i_{x}=i_{x 1}+i_{x 2}+i_{x 3}$, (1) becomes:

$$
L \frac{d i_{x}}{d t}=v_{x 1}+v_{x 2}+v_{x 3}-3 v_{x} .
$$

If we think of $v_{x C O M}$ as the voltage that would be generated from an equivalent single leg, then (2) can be written as

$$
\begin{gathered}
L_{e q} \frac{d i_{x}}{d t}=v_{x C O M}-v_{x} \\
\text { where } L_{e q}=\frac{L}{3} \quad \text { and } \quad v_{x C O M}=\frac{v_{x 1}+v_{x 2}+v_{x 3}}{3} .
\end{gathered}
$$

If the locally-averaging operator is applied to the equations, the switching frequency ripples in the variables are removed and they become continuous.

Considering that the locally-averaged variable $\bar{v}_{x C O M}$ becomes the global reference voltage of the phase, i.e. $\bar{v}_{x C O M}=v_{x R E F}$, (3) can be written as follows:

$$
L_{e q} \frac{d \bar{i}_{x}}{d t}=\bar{v}_{x C O M}-\bar{v}_{x} .
$$

From (4), the averaged equivalent leg of the whole phase can be deduced, as it is shown in Fig. 2.

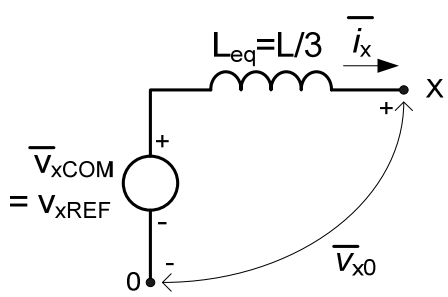

Fig. 2. Averaged equivalent leg

If there were no current balancing control in the system, every leg of a same phase would receive the very same voltage reference, $\bar{v}_{x R E F}$. However, in order to provide a control law for every leg current, each individual voltage is modified as follows:

$$
\bar{v}_{x y}=v_{x R E F}+\Delta \bar{v}_{x y} \text { for } x=\{a, b, c\} \quad y=\{1,2,3\} .
$$

Taking into account that the control variables should not affect the output voltage generated by the leg 
$\left(\bar{v}_{x C O M}=v_{x R E F}\right)$, the control voltages have to meet the following condition:

$$
\Delta \bar{v}_{x}=\Delta \bar{v}_{x 1}+\Delta \bar{v}_{x 2}+\Delta \bar{v}_{x 3}=0 .
$$

Since $\bar{v}_{x C O M}$ becomes unaltered if restriction (6) is applied, $\bar{i}_{x}$ and $\bar{v}_{x 0}$ will also be unaffected by the control variables.

On the other hand, applying the locally-averaging operator to (1):

$$
L \frac{d \bar{i}_{x y}}{d t}=\bar{v}_{x y}-\bar{v}_{x} \quad \text { for } x=\{a, b, c\} \quad y=\{1,2,3\},
$$

and adding the effect of the control variables, the following relationship is obtained:

$$
\begin{gathered}
L \frac{d\left(\bar{i}_{x y}+\Delta \bar{i}_{x y}\right)}{d t}=\bar{v}_{x y}+\Delta \bar{v}_{x y}-\bar{v}_{x}-\Delta \bar{v}_{x} \text { in which } \\
\Delta \bar{i}_{x y}=\bar{i}_{x y}-\frac{\bar{i}_{x}}{3} \text { for } x=\{a, b, c\} \text { and } y=\{1,2,3\} .
\end{gathered}
$$

Comparing (7) and (8), and bearing in mind that $\Delta \bar{v}_{x}=0$ as a consequence of the control restriction given in (6), the following relationship is obtained:

$$
L \frac{d \Delta \bar{i}_{x y}}{d t}=\Delta \bar{v}_{x y} \text { for } x=\{a, b, c\} \quad y=\{1,2,3\} .
$$

Assuming a current imbalance $\Delta \bar{i}_{x y}(k)$ at the instant $k T_{s}$, the necessary voltage to achieve the reference current $\bar{i}_{x} / 3$ can be calculated imposing the condition $\Delta \bar{i}_{x y}(k+1)=0$ to the discrete representation of (9), as follows:

$$
\begin{gathered}
L \frac{\Delta \bar{i}_{x y}(k+1)-\Delta \bar{i}_{x y}(k)}{T_{s}}=\Delta \bar{v}_{x y}(k) \\
\text { with } \Delta \bar{i}_{x y}(k+1)=0 .
\end{gathered}
$$

A timing diagram for this on-line process can be seen in Fig. 3.

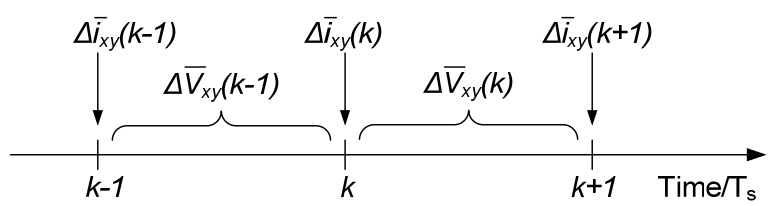

Fig. 3. Time sequence of the sampling process for current balancing

Sometimes, achieving current balance in a single sampling period may not be possible because of the large $\Delta \bar{v}_{x y}$ values required. If so, the control voltages should be limited to their maximum value in order to avoid overmodulation. Condition (6) has always to be satisfied, even when this restriction applies, to avoid distortion in the global output phase voltage.

\section{The Interleaving Technique}

The interleaving technique is applied to the system shown in Fig. 1 to achieve an apparent switching frequency $n$ times higher that the individual switching frequency of each leg $\left(f_{s}=n \cdot f_{s w}=n / T_{s w}\right)$.

When operating with a carrier-based modulation strategy, this is achieved by using an $n$ number of shifted carriers. Fig. 4 shows the general $n$-case carriers disposition.

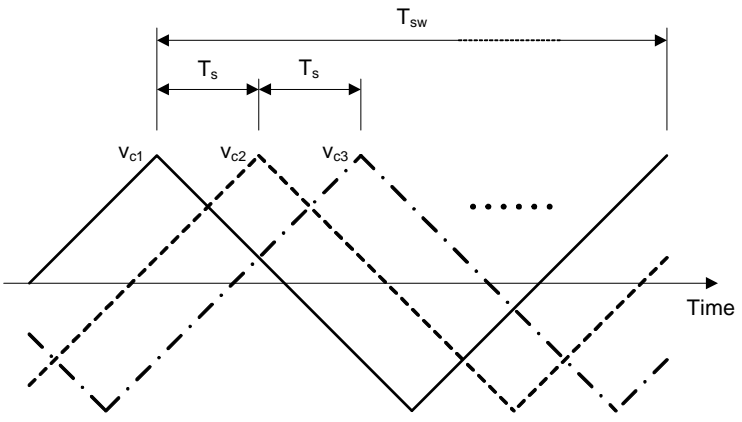

Fig. 4. Phase shift disposition of the carriers for interleaving operation mode

The output current $\left(\bar{i}_{x}\right)$ and the individual current of each leg $\left(\bar{i}_{x y}\right)$ are sensed at the maximum (or minimum) peak of the corresponding carrier $\left(v_{C y}\right)$. The value of the variable $\Delta \bar{v}_{x y}$ is calculated at any sampling period and applied to the particular modulation signal of each leg. Subsequently, the balancing dynamic is as fast as the apparent switching frequency $\left(f_{s}=1 / T_{s}\right)$.

\section{Control strategy.}

The control strategy used in this grid-connected system is a VOC. A short description of this type of control is presented next.

From the d-q model of a grid-connected system [10],[11], the voltage references for the converter can be given by

$$
\begin{gathered}
v_{\text {dref }}=e_{d}-\omega L_{t} i_{q}+\Delta v_{d} \quad \text { and } \\
v_{\text {qref }}=e_{q}+\omega L_{t} i_{d}+\Delta v_{q},
\end{gathered}
$$

where,

$$
\begin{gathered}
\Delta v_{d}=L_{t} \frac{d i_{d}}{d t}+R_{t} i_{d} \quad \text { and } \\
\Delta v_{q}=L_{t} \frac{d i_{q}}{d t}+R_{t} i_{q} .
\end{gathered}
$$

From (11), the control scheme shown in Fig. 5 can be deduced. In this scheme, the positive-sequence angle of the grid voltages $\left(\theta_{d}\right)$ is firstly detected. Then, it is used 


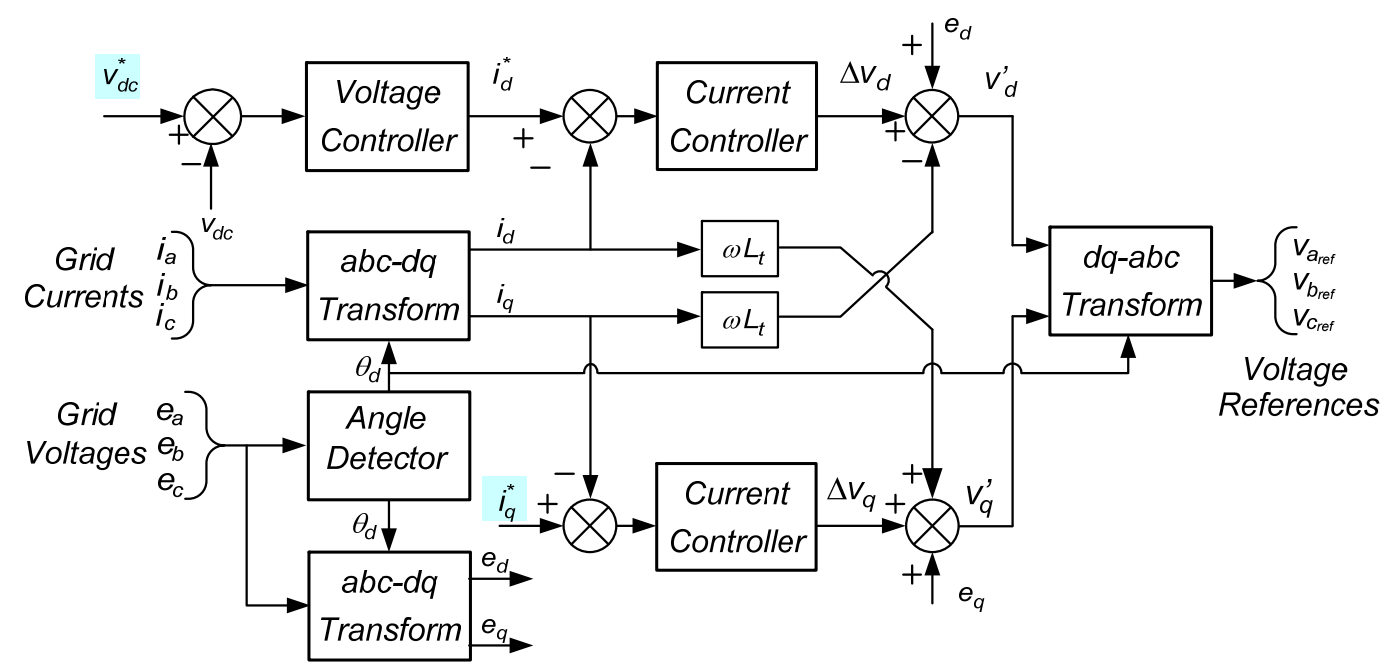

Fig. 5. Control scheme for the dc-link voltage and the grid currents.

for the $d-q$ transformations of the grid currents and voltages. As a consequence of such synchronization, the grid component $e_{q}$ becomes zero when operating under balanced and undistorted grid voltages. Besides, the $d$ and $q$ current components will define active and reactive power, respectively [11].

This control diagram has three loops: an external loop to control the dc-link voltage $\left(v_{d c}\right)$ and two internal ones to regulate the $d$ and $q$ current components.

The controller provides the voltages $\Delta v_{d}$ and $\Delta v_{q}$ that, applied to the equivalent grid impedances, will impose the desired grid currents. In order to obtain the voltage references for the converter, $v_{\text {dref }}$ and $v_{\text {qref }}$, two additional kinds of terms need to be added. One kind of them is added to cancel the crossing influence between the two current components. The other ones are the transformed grid voltages, $e_{\mathrm{d}}$ and $e_{\mathrm{q}}$, although the latter will usually be zero.

In order to achieve unity power factor, the reactive current reference $\left(i_{q}^{*}\right)$ is normally zero for many applications. However, some regulations require imposing a value different from zero during particular circumstances such as grid voltage sags. Under such conditions, specific regulations define the amount of reactive power that should be injected into the electrical grid. This is the case of the P.O. 12.3 in Spain that indicates the conditions required to keep wind turbines connected to the grid throughout a voltage sag process.

\section{Simulation and experimental results}

A model of the grid-connected system with the proposed controllers has been developed on MATLABSIMULINK environment. The main parameters of the model are the following: grid voltages $380 \mathrm{~V}-50 \mathrm{~Hz}$; grid inductors $L_{g}=1 \mathrm{mH}$; leg inductors $L=10 \mathrm{mH}$ with an internal resistance of $R=0.05 \Omega$; dc-link capacitor
$C=2,200 \mu \mathrm{F}$; dc-link reference voltage $V_{d c}^{*}=1000 \mathrm{~V}$; dclink input current $I_{d c}=10 \mathrm{~A}$; carrier frequency $f_{s w}=5 \mathrm{kHz}$; and reference reactive current $i_{q}^{*}=0$.

Fig. 6(a) shows the system starting with the balancing control disconnected. One can see that the currents are not equal and therefore some legs carry more current than the others. The balancing control is activated at the instant $t=38 \mathrm{~ms}$. Observe that the three currents are quickly balanced and the legs carry similar current values henceforth. Thus, similar power losses would be produced in all the transistors of the converter. Additionally, the leg currents contain much more ripple than the output phase currents due to the interleaving effect.

In order to test the reactive current control, Fig. 6(b) shows the phase- $a$ leg and grid currents, and the grid voltage of that phase. At the beginning of the process the reactive current reference is zero but it is changed at the instant of $45 \mathrm{~ms}$, in which it becomes $I_{q}^{*}=-20 \mathrm{~A}$. It can be noted that the relative phase of the grid current changes and it is no longer in phase with the corresponding grid voltage.

Fig. 6(c) shows the equivalent voltage of phase $a$, i. e. $v_{a C O M}=\left(v_{a 1}+v_{a 2}+v_{a 3}\right) / 3$. It can be seen that the equivalent voltage takes four levels as it does in a multilevel converter. This figure also shows the dc-link voltage $\left(v_{d c} / 2\right)$ which is regulated to its reference.

As a first stage of the experimental checking on this study, a single-phase laboratory prototype has been built. The converter has two parallel-connected legs and operates over a passive load. Therefore, since there is no grid connection, the VCO is not implemented, but only the modulation and the proposed current balancing strategy. The main parameters are: $V_{d c}=50 \mathrm{~V}, m_{a}=0.7$, $f=50 \mathrm{~Hz}, f_{s w}=5 \mathrm{kHz}, f_{s}=10 \mathrm{kHz}, L=6 \mathrm{mH}$ with an internal resistance of $R_{p}=0.54 \Omega$, and $R_{\text {load }}=10 \Omega$. 


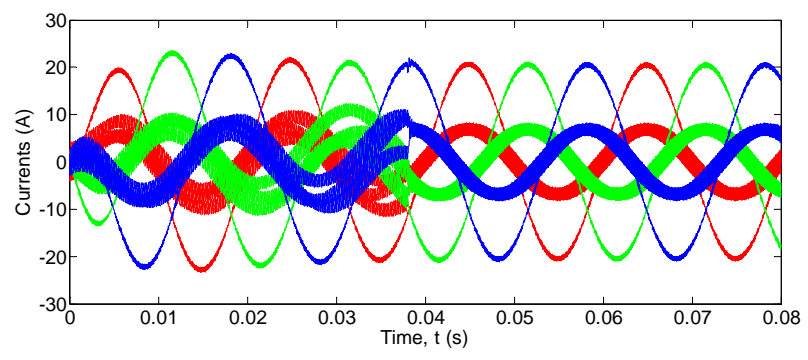

(a)

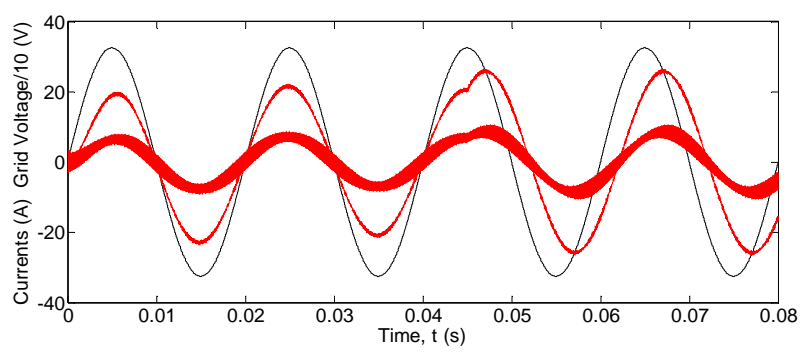

(b)

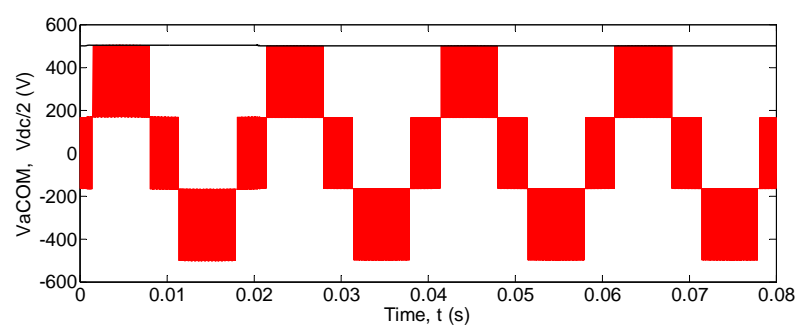

(c)

Fig. 6. Simulation results of the grid-connected system. (a) leg and phase currents with balancing control activation; (b) leg and phase currents and grid voltage with reactive current reference change; and (c) dc-link voltage and equivalent phase voltage $\left(v_{a C O M}\right)$.

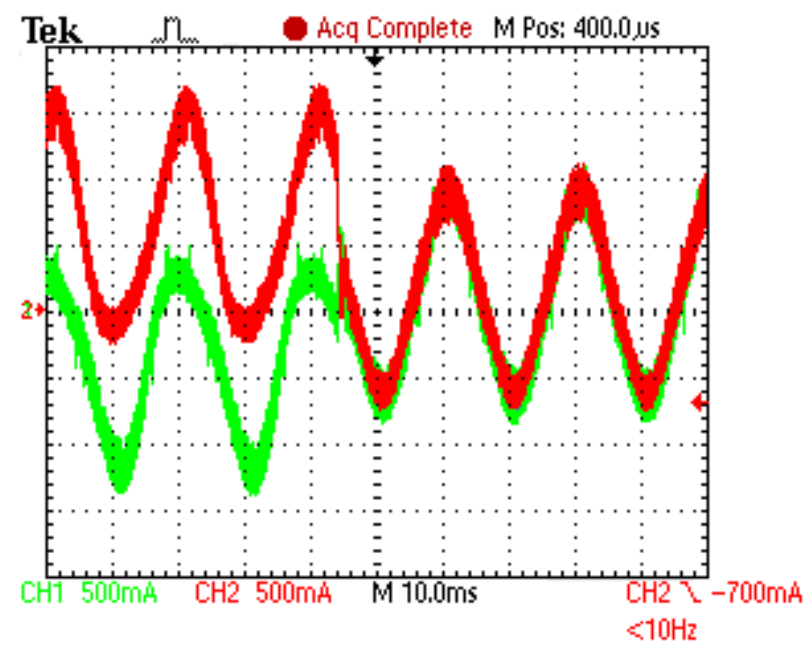

Fig. 7. Experimental results. Continuous current imbalance produced by a small dc voltage difference between the legs and activation of the compensator.

On Fig. 7, a low dc-voltage has been added to the output of one leg by means of a battery. This process emulates the case of different voltage drops in the transistors of the legs. Consequently, the system starts with a permanent current imbalance. When the balancing control is activated, the currents are balanced almost instantaneously.

\section{Conclusion}

Each phase of the grid-connected system studied in this paper is made up of three legs connected in parallel through inductors. The current balancing strategy implemented can achieve evenly shared currents among the legs with very fast dynamics. A VOC loop is able to regulate the dc-link voltage and to control the reactive current delivered to the grid.

This system has special interest for applications such as distributed generation, energy storage, and power quality devices. The particularity of having more than one leg per phase allows not only to increase the rated power of the converter, but also to improve fault tolerance capability. In the case of failing one switch, the corresponding leg could be isolated and the system would keep on operating with a reduction of the maximum output current and power.

\section{Acknowledgement}

This work was supported by the Ministerio de Ciencia y Tecnologia of Spain under Projects ENE2007-67033C03-01 and ENE2007-67033-C03-03, the Department d'Universitats Recerca i Societat de la Informació of the Generalitat de Catalunya, the Basque Country Government, and the Torres Quevedo Program.

\section{References}

[1] W. Hu, Y. Wang, W. Yao, J. Wu, H. Zhang, and Z. Wang: "An efficient experimental method for high power direct drive wind energy conversion systems," in Proc. IEEE Power Electronics Specialists Conference (PESC), 15-19 June 2008, Rhodes, Greece, pp. 3955-3959.

[2] Z. Qiu and G. Chen, "Study and Design of Grid Connected Inverter for $2 \mathrm{MW}$ Wind Turbine," in Proc. IEEE Industry Applications Conference (IAS), 23-27 Sept. 2007, New Orleans, LA, USA, pp. 165-170.

[3] C. Wen, J. Li, X. Zhu, and H. Xu, "Research on circulation of parallel three-phase converters in MW wind power system," in Proc. IEEE Power Electronics and Intelligent Transportation System (PEITS), 2-3 Aug. 2008, Guangzhou, China, pp. 349-354.

[4] I.W. Jaskulski, H. Pinheiro, and L. Mariotto, "Multi-Leg Voltage Source Converter for Grid Connected Wind Turbines," in Proc. Clean Electrical Power (ICCEP), 21-23 May 2007, Capri, Italy, pp. 229-235.

[5] L. Jian-lin, H. Shu-ju, L. Mei, Z. Ying, K. De-guo, and X. Hong-hua, "Research on the application of parallel backto-back PWM converter on direct-drive wind power system," in Proc. Electric Utility Deregulation and Restructuring and Power Technologies (DRPT), 6-9 April 2008, Nanjuing, China, pp. 2504-2508.

[6] V. Agarwal and D.V.M.M. Krishna, "Statistical Approach to Robust Design of Control Schemes for Series or Parallel Connected Power Devices," EPE Journal, Vol. 19, No 3, Sept. 2009, pp. 15-21. 
[7] J.C. Le Claire and G. Lembrouck, "A simple feedback for parallel operation of current controlled inverters involved in UPS," in Proc. European Conference on Power Electronics and Applications (EPE), 2-5 Sept. 2007, Aalborg, Denmark.

[8] S. Fukuda and K. Matsushita, "A control method for parallel-connected multiple inverter systems," in Proc. IEEE Power Electronics and Variable Speed Drives, 21-23 Sep 1998, London, UK, pp. 175-180.

[9] J. Pou, J. Zaragoza, G. Capellá, I. Gabiola, S. Ceballos, and E. Robles, "Current Balancing Strategy in ParallelConnected Legs of Power Inverters," accepted for publication in the EPE Journal.

[10] M. Molinas, B. Naess, W. Gullvik, and T. Undeland, "Cage Induction Generators for Wind Turbines with Power Electronics Converters in the Light of the New Grid Codes," in Proc. European Conference on Power Electronics and Applications (EPE'05), Dresden, Germany, 11-14 Sep. 2005.

[11] J. Pou, E. Robles, S. Ceballos, J. Zaragoza, A. Arias, and P. Ibañez, "Control of Back-to-Back-Connected NeutralPoint-Clamped Converters in Wind Mill Applications," in Proc. European Conference on Power Electronics and Applications (EPE'07), Aalborg, Denmark, 2-5 Sep. 2007. 